K. Aomoto

Nagoya Math. J.

Vol. 68 (1977), 1-16

\title{
ANALYTIC STRUCTURE OF SCHLÄFLI FUNCTION
}

\author{
KAZUHIKO AOMOTO
}

\section{§1. Introduction}

In this note it is shown that Schläfi function can be simply expressed in terms of hyperlogarithmic functions, namely iterated integrals of forms with logarithmic poles in the sense of K. T. Chen (Theorem 1). It is also discussed the relation between Schläfl function and hypergeometric ones of Mellin-Sato type (Theorem 2). From a combinatorial point of view the structure of hyperlogarithmic functions seem very interesting just as the dilog $\int_{0}^{x} \log (1-x) / x d x$ (so-called Abel-Rogers function) has played a crucial part in Gelfand-Gabriev-Losik's formula of 1st Pontrjagin classes. See also [3].

The author would like to express his sincere gratitude to Prof. S. S. Chern who has pointed out to the author his interest in this subject and has communicated some references, and to the author's colleagues too for their stimulating discussions.

\section{§2. Gauss-Bonnet theorem}

Let $S^{n}$ be a $n$ dimensional unit sphere in $\boldsymbol{R}^{n+1}$ with the standard metric and $S_{1}, S_{2}, \cdots, S_{n+1}$ be $(n+1)$ hyperplanes in $\boldsymbol{R}^{n+1}$ through the origin which are in general position. Let

$$
S_{j}: f_{j}=0
$$

where $f_{j}=\sum_{\nu=1}^{n+1} u_{j_{\nu}} x_{\nu}$ with $\sum_{\nu=1}^{n+1} u_{j_{\nu}}^{2}=1$. The set of all points of $S^{n}$ satisfying the inequalities

$$
f_{1} \geqq 0, \cdots, f_{n+1} \geqq 0
$$

form a $n$ dimensional spherical simplex denoted by $\Delta$. We denote by

Received May 31, 1976. 
$\langle i, j\rangle$ the dihedral angle between $S_{i}$ and $S_{j}$ subtended by $\Delta$. Then $\Delta$ is uniquely determined up to the motion of congruences by the $n(n+1) / 2$ quantities $-\cos \langle i, j\rangle=a_{i j}$ so that the volume $V$ of $\Delta$ can be regarded as an analytic function of the variables $a_{i j}$ of the $n(n+1) / 2$ dimensional complex affine space $\mathfrak{X}$, which is defined by Schläfli's integral on $\Delta$ :

$$
V=\int_{\Delta} \sum_{j=1}^{n+1}(-1)^{j-1} \cdot x_{j} d x_{1} \wedge \cdots \wedge d x_{j-1} \wedge d x_{j+1} \wedge \cdots \wedge d x_{n+1}
$$

and which can also be expressed as

$(2.3)^{\prime} \quad V=\frac{1}{2^{n / 2-1} \cdot \Gamma(n / 2+1)} \int_{f_{1} \geqq 0, \cdots, f_{n+1} \geq 0} e^{-1 / 2\left(x_{1}^{2}+\cdots+x_{n+1}^{2}\right)} d x_{1} \wedge \cdots \wedge d x_{n+1}$.

Let $\Delta\left(\varepsilon_{1} i_{1}, \varepsilon_{2} i_{2}, \cdots, \varepsilon_{p} i_{p}\right)$ or $V\left(\varepsilon_{1} i_{1}, \cdots, \varepsilon_{p} i_{p}\right)\left(1 \leqq p \leqq n+1, \varepsilon_{j}= \pm 1\right)$ denote the chains in $S^{n}$ defined by the inequalities

$$
\varepsilon_{1} f_{i_{1}} \geqq 0, \cdots, \varepsilon_{p} f_{i_{p}} \geqq 0
$$

or the volumes of them respectively. Clearly we have

$$
\left\{\begin{array}{l}
V\left(\varepsilon_{1} i_{1}, \cdots, \varepsilon_{p} i_{p}\right)=V\left(-\varepsilon_{1} i_{1}, \cdots,-\varepsilon_{p} i_{p}\right) \\
V\left(\varepsilon_{1} i_{1}, \cdots, \varepsilon_{p} i_{p}\right)+V\left(\varepsilon_{1} i_{1}, \cdots, \varepsilon_{p-1} i_{p-1},-\varepsilon_{p} i_{p}\right) \\
\quad=V\left(\varepsilon_{1} i_{1}, \cdots, \varepsilon_{p-1} i_{p-1}\right) \quad \text { and } \\
V\left(\varepsilon_{1} i_{1}\right)=1 / 2\left|S^{n}\right|
\end{array}\right.
$$

where $\left|S^{n}\right|$ denotes the volume of $S^{n}$ equal to $2 \pi^{n / 2} / \Gamma(n / 2)$.

The following Gauss-Bonnet theorem is well-known ([8], [11]).

Proposition 1. For odd $n$

$$
\{(n-1) / 2\}\left|S^{n}\right|=\sum_{\nu=2}^{n-1} \sum_{i_{1}<\cdots<i_{\nu}}(-1)^{\nu} V\left(i_{1}, i_{2}, \cdots, i_{\nu}\right)
$$

and for even $n$

$$
\{(n-1) / 2\}\left|S^{n}\right|=\sum_{\nu=2}^{n} \sum_{i_{1}<\cdots<i_{\nu}}(-1)^{\nu} V\left(i_{1}, \cdots, i_{\nu}\right)-2 V(1,2, \cdots, n+1) .
$$

This Proposition simply follows from the following combinatorial lemma.

LEMMA 1. Let $\mu$ be a finitely additive measure on a space $X$ and $U_{1}, U_{2}, \cdots, U_{m}$ be a finite number of measurable subsets of $X$. Then we have 


$$
\mu\left(\bigcap_{j}\left(X-U_{j}\right)\right)=\mu(X)+\sum_{\nu=1}^{m}(-1)^{\nu} \mu\left(U_{i_{1}} \cap \cdots \cap U_{i_{\nu}}\right)
$$

if $\mu(X)<\infty$.

According to this Proposition all the volumes $V\left(\varepsilon_{1} i_{1}, \cdots, \varepsilon_{p} i_{p}\right)$ are expressed as linear combinations of $\left|S^{n}\right|, V(i, j), V\left(i_{1}, i_{2}, i_{3}, i_{4}\right), \cdots, V\left(i_{1}, \cdots\right.$, $i_{2 \nu}$ ) where $2 \nu-1=n$ or $n-1$ according as $n$ is odd or even.

\section{§3. Application of Schläfli's formula}

We denote by $D\left(\begin{array}{l}i_{1} i_{2} \cdots i_{p} \\ j_{1} j_{2} \cdots j_{p}\end{array}\right)$ the subdeterminant of the symmetric matrix $A$

$$
A=\left(\begin{array}{ccccc}
1 & a_{12} & \cdots & & a_{1, n+1} \\
a_{21} & 1 & \cdots & & a_{2, n+1} \\
\vdots & & \ddots & & \vdots \\
& & 1 & a_{n, n+1} \\
a_{n+1,1} & & \cdots & a_{n+1, n} & 1
\end{array}\right)
$$

consisting of $i_{1}, \cdots, i_{p}$ th. lines and $j_{1}, \cdots, j_{p}$ th. columns. In particular we shall abreviate $D\left(\begin{array}{c}i_{1} i_{2} \cdots i_{p} \\ i_{1} i_{2} \cdots i_{p}\end{array}\right)$ by $D\left(i_{1}, \cdots, i_{p}\right)$. The matrix $A$ defines a spherical simplex $\Delta$ if and only if $A$ is positive definite. In such a case Hadamard's inequality implies

$$
D\left(i_{1}, \cdots, i_{p}\right) \geqq D\left(j_{1}, \cdots, j_{q}\right)
$$

if $\left(i_{1}, \cdots, i_{p}\right) \subset\left(j_{1}, \cdots, j_{q}\right)$. We denote by $E$ the identity matrix where $\langle i, j\rangle$ are all equal to $\pi / 2$.

Notation. We denote by $I$ a subset of indices $\left\{i_{1}, i_{2}, \cdots, i_{p}\right\}$ of $\{1,2, \cdots, n+1\}$ different from each other and by $I$ its length $p$.

Let $\Delta^{*}\left(i_{1}, \cdots, i_{p}\right)$ be a $(n-p)$ dimensional subsimplex of $\Delta$ contained in the intersection $S_{i_{1} i_{2} \cdots i_{p}}$ of $S^{n}$ and the hyperplanes $f_{i_{1}}=0, \cdots, f_{i_{p}}=0$. We denote by $V^{*}\left(i_{1}, \cdots, i_{p}\right)$ the $(n-p)$ dimensional volume of $\Delta^{*}\left(i_{1}, \cdots, i_{p}\right)$. Then Schläfli's fundamental equality can be stated as follows:

SCHLÄFLI'S FORMULA.

$$
d V=\sum_{i<j} V^{*}(i, j) d\langle i, j\rangle
$$


Proof. [15] or [10] p. 337-p. 340.

This also implies the following:

$$
d V^{*}(I)=\sum_{j_{1}<j_{2}, I \cap\left(j_{1}, j_{2}\right)=\varnothing} V^{*}\left(I,\left(j_{1}, j_{2}\right)\right) d\left\langle\begin{array}{c}
I \\
j_{1}, j_{2}
\end{array}\right\rangle
$$

where $\left\langle\begin{array}{c}I \\ j_{1}, j_{2}\end{array}\right\rangle$ denotes the dihedral angle between $S_{j_{1}}$ and $S_{j_{2}}$ subtended by $\Delta^{*}(I)$ in the $(n-p)$ dimensional sphere $S_{I}$.

From now on we shall assume $n$ equal to odd $2 \nu-1$. Let $T$ be a lower triangular matrix:

$$
T=\left(\begin{array}{cccc}
1 & & & 0 \\
t_{21} & t_{22} & & \\
\vdots & \vdots & \ddots & \\
t_{n+1,1} & t_{n+1,2} & \cdots & t_{n+1, n+1}
\end{array}\right)
$$

such that $t_{22}>0, \cdots, t_{n+1, n+1}>0$ and $T \cdot{ }^{t} T=A . \quad T$ is uniquely determined by $A$ and we have

$$
\langle 1,2\rangle=1 / 2 i \log \left(\frac{-t_{21}+i t_{22}}{-t_{21}-i t_{22}}\right)
$$

The lower triangular matrix $T_{12}$ corresponding to $A^{*}(1,2)$ is equal to

$$
\left(\begin{array}{cccc}
1 & & & 0 \\
\lambda_{4} t_{43} & \lambda_{4} t_{44} & & \\
\vdots & & \ddots & \\
\lambda_{n+1} t_{n+1,3} & \lambda_{n+1} t_{n+1,4} & \cdots & \lambda_{n+1} t_{n+1, n+1}
\end{array}\right\}
$$

where $\lambda_{j}$ denotes $1 / \sqrt{1+t_{j 3}^{2}+\cdots+t_{j j}^{2}}$ for $4 \leqq j \leqq n+1$. Therefore by induction we have

$$
\left\langle\begin{array}{l}
12 \\
34
\end{array}\right\rangle=1 / 2 i \log \left(\frac{-t_{43}+i t_{44}}{-t_{43}-i t_{44}}\right)
$$

or more generally

$$
\left\langle\begin{array}{c}
12 \cdots 2 \mu-32 \mu-2 \\
2 \mu-1,2 \mu
\end{array}\right\rangle=1 / 2 i \log \left(\frac{-t_{2 \mu, 2 \mu-1}+i t_{2 \mu, 2 \mu}}{-t_{2 \mu, 2 \mu-1}-i t_{2 \mu, 2 \mu}}\right)
$$

for $0 \leqq \mu \leqq \nu-1$. On the other hand a simple calculation shows that $t_{i, i-1} / t_{i, i}$ is equal to 
$D\left(\begin{array}{l}12 \cdots i-2, i-1 \\ 12 \cdots i-2, i\end{array}\right) / \sqrt{D(1,2, \cdots, i-2, i-1) D(1,2, \cdots, i-2, i-1, i)}$

so that (3.9) is equal to

$1 / 2 i \log \left(\frac{-D\left(\begin{array}{l}12 \cdots 2 \mu-22 \mu-1 \\ 12 \cdots 2 \mu-22 \mu\end{array}\right)+i \sqrt{D(1,2, \cdots, 2 \mu-2) D(1,2, \cdots, 2 \mu)}}{-D\left(\begin{array}{l}12 \cdots 2 \mu-22 \mu-1 \\ 12 \cdots 2 \mu-22 \mu\end{array}\right)-i \sqrt{D(1,2, \cdots, 2 \mu-2) D(1,2, \cdots, 2 \mu)}}\right)$.

Notation. If $I$ and $J$ are two subsets of indices $I=\left(i_{1}, \cdots, i_{p}\right)$ and $J=\left(i_{1}, i_{2}, \cdots, i_{p}, i_{p+1}, i_{p+2}\right)$, then we denote by $\omega\left(\begin{array}{l}I \\ J\end{array}\right)$ the 1 -form defined by

$$
1 / 2 i d \log \left(\frac{-D\left(\begin{array}{l}
i_{1} i_{2} \cdots i_{p} i_{p+1} \\
i_{1} i_{2} \cdots i_{p} i_{p+2}
\end{array}\right)+i \sqrt{D(I) D(J)}}{-D\left(\begin{array}{l}
i_{1} i_{2} \cdots i_{p} i_{p+1} \\
i_{1} i_{2} \cdots i_{p} i_{p+2}
\end{array}\right)-i \sqrt{D(I) D(J)}}\right) .
$$

When $A$ is equal to $E$, namely $\langle i, j\rangle$ are all equal to $\pi / 2, V$ is reduced to $(1 / 2)^{n+1} \cdot\left|S^{n}\right|$. We denote by $\mathfrak{X}_{i_{1} i_{2} \ldots i_{p}}$ the divisor defined by the equation $D\left(i_{1}, i_{2}, \cdots, i_{p}\right)=0$ in $\mathfrak{X}$. Let $\hat{\mathscr{X}}$ be a $2^{\left(2^{n}-1\right)}$-covering of $\mathscr{X}$ ramified over $\mathfrak{X}_{i_{1} i_{2} \cdots i_{2 \mu}}(1 \leqq \mu \leqq \nu)$, uniformizing all the functions $\left.\sqrt{D\left(i_{1}, i_{2}, \cdots, i_{2 \mu}\right.}\right)$, and $\pi$ be the natural projection from $\hat{X}$ onto $\mathfrak{X}$. If $p$ is even, the form $\omega\left(\begin{array}{l}I \\ J\end{array}\right)$ of (3.11) is well-defined 1-form on $\hat{\mathscr{X}}$ which has logarithmic poles along $\pi^{-1}\left(\mathfrak{X}_{i_{1} i_{2} \ldots i_{p} i_{p+1}}\right)$ or $\pi^{-1}\left(\mathfrak{i}_{i_{1} i_{2} \ldots i_{p} i_{p+2}}\right)$ in view of Jacobi's identity:

$$
\begin{aligned}
& D\left(i_{1} \cdots i_{p}\right) D\left(i_{1} \cdots i_{p} i_{p+1} i_{p+2}\right) \\
& \quad=D\left(i_{1} \cdots i_{p} i_{p+1}\right) D\left(i_{1} \cdots i_{p} i_{p+2}\right)-D\left(\begin{array}{l}
i_{1} \cdots i_{p} i_{p+1} \\
i_{1} \cdots i_{p} i_{p+2}
\end{array}\right)^{2} .
\end{aligned}
$$

Definition. Let $\Omega(M ; p, q)$ be the space of continuous paths from a point $p$ to a point $q$ in a differentiable manifold $M$, and $\omega_{1}, \omega_{2}, \cdots, \omega_{m}$ a finite number of differential 1-forms on $M$. Let $\gamma$ be a path of $\Omega(M$; $p, q)$ namely a differentiable function $\varphi:[0,1] \rightarrow M$ such that $\varphi(0)=p$ and $\varphi(1)=q$. Let $f_{j}(t) d t$ be the pull-back of each $\omega_{j}$ by $\varphi$. According to $K$. T. Chen (see [4]) we consider the following integral

$$
\int_{0}^{1} f_{1}\left(t_{1}\right) d t_{1} \int_{0}^{t_{1}} f_{2}\left(t_{2}\right) d t_{2} \cdots \int_{0}^{t_{m-1}} f_{m}\left(t_{m}\right) d t_{m}
$$


which will be called "iterated integral of order $m$ " and denoted by

$$
\int_{r} \omega_{1} \circ \omega_{2} \circ \cdots \circ \omega_{m}
$$

Now by (3.3), (3.4), (3.10) and (3.11) we can conclude the following:

THEOREM 1. For odd $n, V$ is expressed in terms of iterated integrals of forms of logarithmic poles $\omega\left(\begin{array}{l}I \\ J\end{array}\right)$ on $\hat{\mathscr{X}}$ :

$$
V=\sum_{\left(I_{0}, I_{1}, \cdots, I_{\nu}\right)} \sum_{\sigma=0}^{\nu} \int_{E}^{A} \omega\left(\begin{array}{c}
I_{0} \\
I_{1}
\end{array}\right) \circ \omega\left(\begin{array}{c}
I_{1} \\
I_{2}
\end{array}\right) \circ \cdots \circ \omega\left(\begin{array}{c}
I_{\sigma-1} \\
I_{\sigma}
\end{array}\right) \cdot \frac{\left|S^{n-2 \sigma}\right|}{2^{n+1-2 \sigma}}
$$

where we put $\left|S^{-1}\right|=1$ and $\left(I_{0}, I_{1}, \cdots, I_{\nu}\right)$ run through all families of subsets of indices such that (i) $\left|I_{0}\right|=0,\left|I_{1}\right|=2, \cdots,\left|I_{\nu}\right|=2 \nu$ and (ii) $I_{0}$ $=\emptyset \subset I_{1} \subset I_{2} \subset \cdots \subset I_{\nu}$. The above iterated integrals are done on each path from $E$ to $A$ in $\hat{\mathscr{X}}$.

Remark. The right hand side of (3.15) depends only on homotopy classes of paths provided $A$ is fixed. In fact Chen's formula of the exterior differentiation of iterated integrals show (see Proposition 4.1.2 in [4])

$$
\begin{aligned}
d \sum_{\left(I_{0}, I_{1}, \cdots, I_{\sigma}\right)} \int \omega\left(\begin{array}{c}
I_{0} \\
I_{1}
\end{array}\right) \circ \omega\left(\begin{array}{c}
I_{1} \\
I_{2}
\end{array}\right) \circ \cdots \circ \omega\left(\begin{array}{c}
I_{\sigma-1} \\
I_{\sigma}
\end{array}\right) \\
\quad=\sum_{\left(I_{0}, I_{1}, \cdots, I_{\sigma}\right)} \int \sum_{\tau=1}^{\sigma-1}(-1)^{\tau} \omega\left(\begin{array}{c}
I_{0} \\
I_{1}
\end{array}\right) \circ \cdots \circ \omega\left(\begin{array}{c}
I_{\tau-1} \\
I_{\tau}
\end{array}\right) \wedge \omega\left(\begin{array}{c}
I_{\tau} \\
I_{\tau+1}
\end{array}\right) \circ \cdots \circ \omega\left(\begin{array}{c}
I_{\sigma-1} \\
I_{\sigma}
\end{array}\right)
\end{aligned}
$$

where $\left(I_{0}, I_{1}, \cdots, I_{\sigma-1}\right)$ run through all the subsets of indices such that $\left|I_{0}\right|=0,\left|I_{1}\right|=2, \cdots, I_{\sigma}=2 \sigma$ and $I_{0} \subset I_{1} \subset \cdots \subset I_{\sigma-1} \subset I_{\sigma}, I_{\sigma}$ being fixed. This vanishes in view of the following identities:

$$
\sum_{\substack{I \subset K \subset J \\
|K|=|I|+2}} \omega\left(\begin{array}{l}
I \\
K
\end{array}\right) \wedge \omega\left(\begin{array}{l}
K \\
J
\end{array}\right)=0
$$

for any subsets of indices $I$ and $J$ such that $|I|+4=|J|$, which can be proved by a direct calculation.

COROLLARY OF THEOREM 1. The monodromy of the many valued function $V$ on $\hat{\mathscr{X}}$ is contained in a unipotent subgroup of upper triangular matrices.

Proof. This follows from a general theory of iterated integrals 
(see [4] p. 222). In our situation the variation of $V$ along an arbitrary loop on $\hat{\mathscr{X}}-\underset{\substack{i_{1}<\cdots<i_{2 \mu} \\ 1 \leqslant \mu \leqslant \nu}}{\bigcup} \pi^{-1}\left(\mathfrak{X}_{i_{1} \cdots i_{2} \mu}\right)$ can be written as a linear combination of the iterated integrals

$$
\sum_{I_{1}, \cdots, I_{\sigma-1}} \omega\left(\begin{array}{c}
I_{0} \\
I_{1}
\end{array}\right) \circ \omega\left(\begin{array}{c}
I_{1} \\
I_{2}
\end{array}\right) \circ \cdots \circ \omega\left(\begin{array}{c}
I_{\sigma-1} \\
I_{\sigma}
\end{array}\right)
$$

which is closed on $\Omega$ because of (3.16). This fact can also be proved in a direct way by using a generalized Picard-Lefschetz formula due to $\mathrm{F}$. Pham.

According to $\mathrm{H}$. Poincaré and Lappo-Danilevski we shall call "hyperlogarithmic functions of order $m$ " functions of iterated integrals of $m$ th order of forms with logarithmic poles, so that $V$ is a hyperlogarithmic function of order $\nu$ on $\hat{\mathscr{X}}$.

The volume of a double-rectangular tetrahedron was investigated by H. S. M. Coxeter [6]. By his notations we have $\langle 1,3\rangle=\langle 1,4\rangle=\langle 2,4\rangle=0$, $\langle 1,2\rangle=\pi / 2-\alpha,\langle 2,3\rangle=\beta$ and $\langle 3,4\rangle=\pi / 2-\gamma$. Then $V$ is written as follows :

$$
\begin{aligned}
V-\left|S^{3}\right| / 16= & -\int d \alpha \cdot 1 / 2 i \log \left(\frac{-\sin \gamma \cos \alpha+i \sqrt{D}}{-\sin \gamma \cos \alpha-i \sqrt{D}}\right) \\
& +\int d \beta \cdot 1 / 2 i \log \left(\frac{-\sin \alpha \cos \beta \sin \gamma+i \sin \beta \sqrt{D}}{-\sin \alpha \cos \beta \sin \gamma-i \sin \beta \sqrt{D}}\right) \\
& -\int d \gamma \cdot 1 / 2 i \log \left(\frac{-\sin \alpha \cos \gamma+i \sqrt{D}}{-\sin \alpha \cos \gamma-i \sqrt{D}}\right)
\end{aligned}
$$

which gives the same formula as (4.11) in [6], where $D$ means $D(1,2,3,4)$ $=\cos ^{2} \alpha \cdot \cos ^{2} \gamma-\cos ^{2} \beta$.

\section{§4. Power series expansion of $V$}

The integral (1.3) can also be expressed as follows:

$$
V=(n+1) \int_{\substack{f_{1} \geq 0, \cdots, f_{n+1} \geq 0 \\ 1 \geqq x_{1}^{2}+\cdots+x_{n+1}^{2}}} d x_{1} \wedge \cdots \wedge d x_{n+1}
$$

By change of variables the right hand side is transformed into

$$
(n+1) / D \int_{\substack{1 \geq Q\left(y_{1}, \ldots, y_{n+1}\right) \\ y_{1} \geq 0, \cdots, y_{n+1} \geqq 0}} d y_{1} \wedge \cdots \wedge d y_{n+1}
$$


where $Q$ denotes the quadratic polynomial $\sum_{j=1}^{n+1} y_{j}^{2}+\sum_{i \neq j} b_{i j} y_{i} y_{j}$ with $b_{i j}=b_{j i}$ and $D$ denotes $D(1,2, \cdots, n+1) . \quad b_{i j}$ are determined by the relation:

$$
B=K^{-1} \cdot A^{-1} \cdot K^{-1},
$$

where $B$ denotes the matrix

$$
\left(\begin{array}{cccc}
1 & b_{12} & \cdots & b_{1, n+1} \\
b_{21} & 1 & \cdots & b_{2, n+1} \\
\vdots & & \ddots & \vdots \\
b_{n+1,1} & b_{n+1,2} & \cdots & 1
\end{array}\right)
$$

and $K$ denotes the diagonal matrix with positive elements Diag $\left[\rho_{1}, \cdots, \rho_{n+i}\right]$, $\rho_{i}$ equal to

$$
\sqrt{\frac{D(1, \cdots, i-1, i+1, \cdots, n+1)}{D(1,2, \cdots \cdots \cdots \cdots \cdots \cdots, n+1)}} .
$$

It is easily seen that the correspondence (4.3) is birational on $\hat{\mathscr{X}}$, leaving fixed the divisors $\bigcup_{1 \leqslant \mu \leqslant \nu} \bigcup_{i_{1}<i_{2}<\cdots<i_{2 \mu}} \pi^{-1}\left(\mathfrak{X}_{i_{1} i_{2} \cdots i_{2} \mu}\right)$ or $\bigcup_{1 \leqslant \mu \leqslant \nu} \bigcup_{i_{1} i_{2}<\cdots<i_{2 \mu-1}} \pi^{-1}\left(\mathfrak{X}_{i_{1} i_{2} \cdots i_{2 \mu-1}}\right)$.

Now we are going to prove the following theorem:

THEOREM 2. As a function of the variables $b_{i j}, V$ has a convergent power series expansion at the origin:

$$
\begin{array}{r}
2^{n+1} \cdot D V /(n+1)=\sum_{\sigma_{i j \geqq 0}} \frac{\prod_{i<j}\left(-2 b_{i j}\right)^{\sigma_{i j}}}{\prod_{i<j} \sigma_{i j} !} \\
\cdot \frac{\prod_{k=1}^{n+1} \Gamma\left(\frac{\sigma_{1, k}+\cdots+\sigma_{k-1, k}+\sigma_{k, k+1}+\cdots+\sigma_{k, n+1}}{2}\right)}{\Gamma\left(\frac{n+1}{2}+1\right)}
\end{array}
$$

which is a so-called generalized hypergeometric series. For this kind of functions see Appendix.

To prove Theorem 2 we want to prove a slightly more general theorem by making use of a technic introduced in [1].

THEOREM 2'. The integral

$$
\varphi=\int_{\substack{1 \geq Q \\ y_{1} \geqq 0, \cdots, y_{n+1} \geqq 0}}(1-Q)^{\lambda_{0}} y_{1}^{\lambda_{1}} \cdot y_{2}^{\lambda_{2}} \cdots y_{n+1}^{\lambda_{n}+1} d y_{1} \wedge \cdots \wedge d y_{n+1}
$$


for $\lambda_{0} \geqq 0, \lambda_{1} \geqq 0, \cdots, \lambda_{n+1} \geqq 0$ has a convergent power series expansion near the origin:

$$
\begin{aligned}
2^{n+1} \varphi & =\frac{\prod_{i<j}\left(-2 b_{i j}\right)^{o_{i j}}}{\prod_{i<j} \sigma_{i j} !} \\
& \frac{\prod_{k=1}^{n+1} \Gamma\left(\frac{\sigma_{1 k}+\cdots+\sigma_{k-1, k}+\sigma_{k, k+1}+\cdots+\sigma_{k, n+1}+\lambda_{k}+1}{2}\right) \Gamma\left(\lambda_{0}+1\right)}{\Gamma\left(\frac{\lambda_{1}+\cdots+\lambda_{n+1}+n+1}{2}+\lambda_{0}+1\right)}
\end{aligned}
$$

To prove (4.7) we need

LEMMA 2. If $\lambda_{0}, \lambda_{1}, \cdots, \lambda_{n+1}$ are all sufficiently large,

$$
\begin{aligned}
\int_{\substack{1 \geq Q, \ldots, y_{n} \geq 0 \\
y_{1} \geq 0, \cdots, y_{n+1} \geq 0}} y_{1}^{\lambda_{1}} \cdot y_{2}^{\lambda_{2}} \cdots y_{n+1}^{\lambda_{n}+1}(1-Q)^{\lambda_{0}-1} d y_{1} \wedge \cdots \wedge d y_{n+1} \\
=\left(n+1+2 \lambda_{0}+\lambda_{1}+\cdots+\lambda_{n+1}\right) / 2 \lambda_{0} \\
\cdot \int y_{1}^{\lambda_{1}} \cdot y_{2}^{\lambda_{2}} \cdots y_{n+1}^{\lambda_{n}+1}(1-Q)^{\lambda_{0}} d y_{1} \wedge \cdots \wedge d y_{n+1} .
\end{aligned}
$$

Proof. We have by exterior differentiation

$$
\begin{aligned}
& d\left(-\frac{(1-Q)^{\lambda_{0}} y_{1}^{\lambda_{1}} \cdots y_{n+1}^{\lambda_{n}+1}}{2 \lambda_{0}} \sum(-1)^{j-1} y_{j} d y_{1} \wedge \cdots\right. \\
&\left.\wedge d y_{j-1} \wedge d y_{j+1} \wedge \cdots \wedge d y_{n+1}\right) \\
&=\left\{\frac{1}{(1-Q)}-\frac{1}{2 \lambda_{0}}\left(\sum_{1}^{n+1} \lambda_{j}+2 \lambda_{0}+n+1\right)\right\}(1-Q)^{\lambda_{0}} y_{1}^{\lambda_{1}} \cdots y_{n+1}^{\lambda_{n+1}} \\
& \cdot d y_{1} \wedge \cdots \wedge d y_{n+1} .
\end{aligned}
$$

Integrating both sides we get Lemma 2 .

Proof of Theorem 2. For sufficiently large $\lambda_{0}, \lambda_{1}, \cdots, \lambda_{n+1}$ we have

$$
\begin{aligned}
(-1)^{\sigma} \frac{\partial^{\sigma} \varphi}{\partial b_{i_{1} i_{2}} \cdots \partial b_{i_{2 \sigma-1} i_{2 \sigma}}}= & 2^{\sigma} \lambda_{0}\left(\lambda_{0}-1\right) \cdots\left(\lambda_{0}-\sigma+1\right) \\
= & \int_{\substack{1 \geq Q \\
y_{1} \geqq 0, \cdots, y_{n+1} \geqq 0}}(1-Q)^{\lambda_{0}-\sigma} \cdot y_{1}^{\lambda_{1}} \cdots y_{n+1}^{\lambda_{n+1}} \cdot y_{i_{1}} \cdot y_{i_{2}} \\
& \cdots y_{i_{2 \sigma-1}} \cdot y_{i_{2 \sigma}} d y_{1} \wedge \cdots \wedge d y_{n+1} .
\end{aligned}
$$

According to Lemma 2 the right hand side is equal to 


$$
\begin{aligned}
& \prod_{k=1}^{\infty}\left(\sum_{1}^{n+1} \lambda_{j}+2 \lambda_{0}+n+1+2 \sigma-2(k-1)\right) \\
& \int_{\substack{1 \geq Q, \ldots, y_{n+1} \geqq 0 \\
y_{1} \geq 0, \cdots, y_{n+1}}}(1-Q)^{\lambda_{0}} y_{1}^{\lambda_{1}} \cdots y_{n+1}^{\lambda_{n+1}} y_{i_{1}} y_{i_{2}} \cdots y_{i_{2 \sigma-1}} y_{i_{2 \sigma}} d y_{1} \wedge \cdots \wedge d y_{n+1}
\end{aligned}
$$

When $b_{i j}$ are all zero, then $\varphi$ is reduced to

$$
\frac{\prod_{k=1}^{n+1} \Gamma\left(\frac{\lambda_{k}+1}{2}\right) \Gamma\left(\lambda_{0}+1\right)}{2^{n} \Gamma\left(\frac{\lambda_{1}+\cdots+\lambda_{n+1}+n+1}{2}+\lambda_{0}+1\right)} .
$$

Theorem $2^{\prime}$ follows from $(4.8) \sim(4.11)$, because the convergence of the power series (4.7) is obvious. The proof is complete.

Now we want to express $V$ as power series expansion of the variables $t_{i j}$ similar to (3.5) so that

$$
\left\{\begin{array}{l}
f_{1}=x_{1}, \\
f_{2}=t_{21} \cdot x_{1}+x_{2} \\
\cdots \\
f_{n+1}=t_{n+1,1} x_{1}+\cdots+t_{n+1, n} x_{n}+x_{n+1} .
\end{array}\right.
$$

We consider the integral $V\left(\lambda_{1}, \lambda_{2}, \cdots, \lambda_{n+1}\right)$ :

$$
\int_{f_{1} \geqq 0, \cdots, f_{n+1} \geqq 0} f_{1}^{\lambda_{1}} \cdot f_{2}^{\lambda_{2}} \cdots f_{n+1}^{\lambda_{n+1}} d x_{1} \wedge d x_{2} \wedge \cdots \wedge d x_{n+1} .
$$

Then for large $\lambda_{1}, \lambda_{2}, \cdots, \lambda_{n+1}$ we have

$$
\begin{aligned}
& \frac{\partial^{\sigma} V\left(\lambda_{1}, \lambda_{2}, \cdots, \lambda_{n+1}\right)}{\left(\partial t_{21}\right)^{\sigma_{21}} \cdots\left(\partial t_{i j}\right)^{\sigma_{i j}} \cdots\left(\partial t_{n+1, n}\right)^{\sigma_{n+1, n}}} \\
& =\int_{f_{1} \geqq 0, \cdots, f_{n+1} \geqq 0} f_{1}^{\lambda_{1}} \cdot f_{2}^{\lambda_{2}-\sigma_{21}} \cdots f_{n+1}^{\lambda_{n}+\sigma_{n+1,1}-\cdots-o_{n+1, n}} \cdot x_{1}^{\sigma_{21}+\cdots+\sigma_{n+1,1}} \\
& \cdot x_{2}^{\sigma_{32}+\cdots+\sigma_{n+1,2}} \cdots x_{n}^{\sigma_{n+1, n}} d x_{1} \wedge \cdots \wedge d x_{n+1} \\
& \cdot \prod_{i=1}^{n+1} \lambda_{i}\left(\lambda_{i}-1\right) \cdots\left(\lambda_{i}-\sigma_{i, 1}-\cdots-\sigma_{i, i-1}\right) \text {. }
\end{aligned}
$$

For all $t_{i j}=0$, the above is reduced to

$$
\frac{\prod_{k=1}^{n+1} \Gamma\left(\frac{\lambda_{k}-\sigma_{k 1}-\cdots-\sigma_{k, k-1}+\sigma_{k+1, k}+\cdots+\sigma_{n+1, k}+1}{2}\right)}{2^{n+1} \Gamma\left(\frac{\lambda_{1}+\lambda_{2}+\cdots+\lambda_{n+1}+n+1}{2}\right)}
$$




$$
\prod_{i=1}^{n+1} \lambda_{i}\left(\lambda_{i}-1\right) \cdots\left(\lambda_{i}-\sigma_{i, 1}-\cdots-\sigma_{i, i-1}+1\right)
$$

so that (4.13) is equal to

$$
\begin{aligned}
& \sum \frac{\prod \Gamma\left(\frac{\lambda_{k}-\sigma_{k 1}-\cdots-\sigma_{k, k-1}+\sigma_{k+1, k}+\cdots+\sigma_{n+1, k}+1}{2}\right)}{2^{n+1} \Gamma\left(\frac{\lambda_{1}+\lambda_{2}+\cdots+\lambda_{n+1}+n+1}{2}\right) \prod_{i>j} \sigma_{i j} !} \\
& \prod \frac{\Gamma\left(\lambda_{i}+1\right)}{\Gamma\left(\lambda_{i}-\sigma_{i, 1}-\cdots-\sigma_{i, i-1}+1\right)} .
\end{aligned}
$$

In particular if $\lambda_{1}=\cdots=\lambda_{n+1}=0$ we have the volume $V$ :

THEOREM 2 .

$$
\begin{aligned}
V= & \operatorname{li} \operatorname{i.m}_{\lambda_{j \rightarrow 0}} V\left(\lambda_{1}, \cdots, \lambda_{n+1}\right) \\
= & \sum_{\sigma_{i j \geqq 0}} \frac{\prod_{k=1}^{n+1} \Gamma\left(\frac{-\sigma_{k, 1}-\cdots-\sigma_{k, k-1}+\sigma_{k+1, k}+\cdots+\sigma_{n+1, k}+1}{2}\right)}{2^{n+1} \Gamma\left(\frac{n+1}{2}\right) \prod_{i>j} \sigma_{i j} ! \prod_{i=2}^{n+1} \Gamma\left(-\sigma_{i 1}-\cdots-\sigma_{i i-1}+1\right)} \\
& \cdot \prod_{i>j}\left(t_{i j}\right)^{\sigma_{i j}}
\end{aligned}
$$

where the quotients

$$
\frac{\Gamma\left(\frac{-\sigma_{k 1}-\cdots-\sigma_{k, k-1}+\sigma_{k+1, k}+\cdots+\sigma_{n+1, k}+1}{2}\right)}{\Gamma\left(-\sigma_{k 1}-\cdots-\sigma_{k, k-1}+1\right)}
$$

have definite values and the right hand side is well-defined. This is also a hypergeometric function.

\section{§5. Hyperbolic case}

Let $H$ be the hyperbolic space form defined by

$$
\left\{\begin{array}{l}
-x_{1}^{2}-\cdots-x_{n}^{2}+x_{n+1}^{2}=1 \\
x_{n+1}>0
\end{array}\right.
$$

with the standard metric. In view of $(2.3)^{\prime}$ the analytic continuation $V_{\theta}$ of $V$ along the path $\left\{\varphi_{\theta}\right\}$ (see (2.1))

$$
\varphi_{\theta}:\left\{\begin{array}{l}
u_{j, n+1} \rightarrow u_{j, n+1}^{\prime}=u_{j, n+1} \cdot e^{\sqrt{-1} \theta} \\
u_{j k} \rightarrow u_{j k}^{\prime}=u_{j k}
\end{array}\right.
$$


from $\theta=0$ to $\theta=\frac{\pi}{2}(1 \leqq j \leqq n+1,1 \leqq k \leqq n)$ can be written as follows :

$$
\begin{aligned}
V_{\pi / 2} /(\sqrt{-1})^{n}= & \frac{1}{2^{n / 2-1} \Gamma\left(\frac{n}{2}+1\right)} \\
& \cdot \int_{f_{1} \geqq 0, \cdots, f_{n+1} \geqq 0} e^{-\frac{1}{2}\left(-x_{1}^{2}-\cdots-x_{n}^{2}+x_{n+1}^{2}\right)} d x_{1} \wedge \cdots \wedge d x_{n} \wedge d x_{n+1} .
\end{aligned}
$$

The second hand side is equal to the volume $V^{\prime}$ of the simplex $\Delta^{\prime}$ in $H$ defined by $f_{1} \geqq 0, \cdots, f_{n+1} \geqq 0$ and with the faces $H_{i}: f_{i}=0$. The dihedral angle between $H_{i}$ and $H_{j}$ subtended by $\Delta^{\prime}$ is equal to (see (2.1))

$$
-\cos \langle i, j\rangle^{\prime}=\frac{\sum_{\nu=1}^{n} u_{i_{\nu}} u_{j_{\nu}}-u_{i n+1} u_{j n+1}}{\sqrt{\sum_{\nu=1}^{n} u_{i \nu}^{2}-u_{i n+1}^{2}} \sqrt{\sum_{\nu=1}^{n} u_{j_{\nu}}^{2}-u_{j n+1}^{2}}}
$$

so that Schläfli formula has the form:

$$
d V^{\prime}=-\sum_{i<j} V^{\prime}(i, j) d\langle i, j\rangle^{\prime}
$$

where $V^{\prime}(i, j)$ means the volume of the $(n-2)$ dimensional subsimplex $\Delta^{*}(i, j)^{\prime}$ defined by $f_{i}=f_{j}=0$.

We denote by $A^{\prime}$ the matrix corresponding to $\langle i, j\rangle^{\prime}$ :

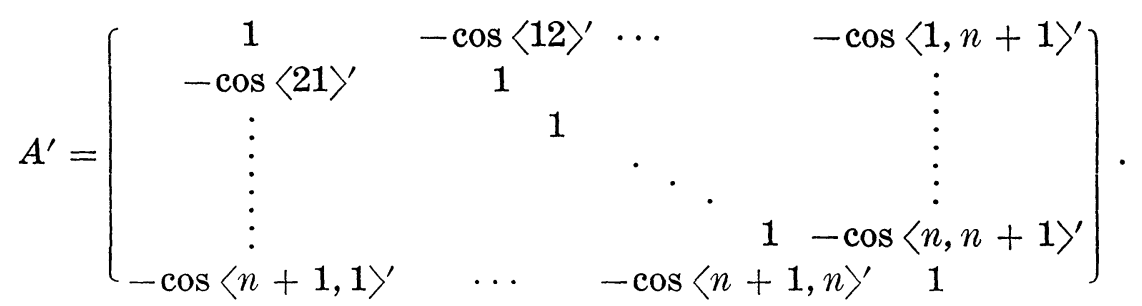

Then Theorem 1 implies the following:

THEOREM 1'. For odd $n$ we have

$$
(\sqrt{-1})^{n} V^{\prime}=\sum_{\left(I_{0}, I_{1}, \ldots, I_{\nu}\right)} \sum_{\sigma=0}^{\nu} \int_{E}^{A^{\prime}} \omega\left(\begin{array}{c}
I_{0} \\
I_{1}
\end{array}\right) \circ \omega\left(\begin{array}{c}
I_{1} \\
I_{2}
\end{array}\right) \circ \cdots \circ \omega\left(\begin{array}{c}
I_{\sigma-1} \\
I_{\sigma}
\end{array}\right) \frac{\left|S^{n-2 \sigma}\right|}{2^{n+1-2 \sigma}}
$$

where the integrals are done on each path from $E$ to $A^{\prime}$ in $\hat{\mathscr{X}}$.

COROLlary. Let $\bar{V}^{\prime}$ be the volume of a hyperbolic simplex $\bar{\Delta}^{\prime}$ corresponding to a fixed point $\bar{A}^{\prime} \in \hat{\mathscr{X}}$. Then $V^{\prime}-\bar{V}^{\prime}$ is equal to a linear combination of the iterated integrals along a path from $\bar{A}^{\prime}$ to $A^{\prime}$ : 


$$
\int_{\bar{A}^{\prime}}^{A^{\prime}} \omega\left(\begin{array}{l}
I_{0} \\
I_{1}
\end{array}\right) \circ \omega\left(\begin{array}{c}
I_{1} \\
I_{2}
\end{array}\right) \circ \cdots \circ \omega\left(\begin{array}{c}
I_{\sigma-1} \\
I_{\sigma}
\end{array}\right) \quad(0 \leqq \sigma \leqq \nu)
$$

where $I_{0}, I_{1}, \cdots, I_{\nu}$ run through all the subsets of indices such that (i) $\left|I_{0}\right|=0,\left|I_{1}\right|=2, \cdots,\left|I_{\nu}\right|=2 \nu$ and (ii) $I_{0}=\emptyset \subset I_{1} \subset \cdots \subset I_{\nu}$.

Proof. This easily follows from Proposition 1.5.1 in [4].

\section{Appendix. Hypergeometric functions of Mellin-Sato type}

We reproduce here briefly Sato's result in [14].

Let $G$ be the group of $m$-product of $C^{*}=C-(0)$ and $X$ be its dual, Hom $\left(G, C^{*}\right)$ which is isomorphic to $Z^{m}$. We denote by $X_{\boldsymbol{C}}$ its complexification. Let $\left\{\chi_{1}, \cdots, \chi_{m}\right\}$ be a basis of $X$ so that any $\omega$ of $X_{c}$ can be written as $\omega=\sum_{j=1}^{m} s_{j} \chi_{j}$ with $\left(s_{1}, \cdots, s_{m}\right) \in C^{m}$.

Notation. For a rational function $f(\nu)$ on $Z$ we denote by $\prod_{\nu=0}^{e-1} f(\nu)$ the product:

$$
\begin{cases}\prod_{\nu=0}^{e-1} f(\nu) & e \geqq 1 \\ 1 / \prod_{\nu=e}^{-1} f(\nu) & e<0 \\ 1 & e=0\end{cases}
$$

Under this situation Sato's fundamental theorem says

THEOREM A, 1 . Each class in the cohomology $H^{1}(X, C(x))$ can be represented by a so-called "b-function"

$$
b_{\chi}(\omega)=\prod_{\kappa=1}^{k}\left\{\prod_{\nu=0}^{e_{\kappa}(x)-1}\left(e_{k}(\omega)+\alpha_{\kappa}+\nu\right)\right\}
$$

where $\alpha_{\kappa}$ denotes a constant and $e_{\kappa}$ a suitable $\mathbf{Q}$-valued linear function on $\boldsymbol{Z}^{m}$.

Let $X_{C}^{*}$ be the dual of $X_{c}$ so that $X_{c}^{*}$ is isomorphic to the Lie algebra corresponding to $G$. For any point $\tau$ of $X_{c}^{*}$ we put $e^{\tau}=t$, where $t=\left(t_{1}, \cdots, t_{m}\right) \in G$. We denote by $t^{x}$ the pairing $e^{\langle\chi, \tau\rangle}$.

Definition. Arbitrary function $u$ on $X_{c}^{*}$ satisfied by the following system of (pseudo) differential equations 


$$
b_{x}\left(t_{1} \frac{\partial}{\partial t_{1}}, \cdots, t_{m} \frac{\partial}{\partial t_{m}}\right) u=t^{-x} \cdot u
$$

for any $\chi \in X$, is called "hypergeometric function of Mellin-Sato type". This system is maximally overdetermined on $X_{c}^{*}$.

LEMmA A, 1. The Mellin transform of a generalized $\Gamma$-function $\hat{u}(\omega)$

$$
\left\{\begin{array}{l}
\hat{u}(\omega)=\prod_{k=1}^{k} \Gamma\left(e_{\boldsymbol{k}}(\omega)+\alpha_{\boldsymbol{k}}\right) \\
u(t)=\int \hat{u}(\omega) t^{\omega} d \omega_{1} \cdots d \omega_{m}
\end{array}\right.
$$

is a hypergeometric function of $M-S$ type if it exists.

Proof. Easy.

THEOREM $2^{\prime} \quad D \cdot V$ is a Mellin transform of $\hat{V}$

$$
\begin{aligned}
\hat{V}= & \prod_{k=1}^{n+1} \Gamma\left(\frac{s_{1 k}+\cdots+s_{k-1, k}+s_{k, k+1}+\cdots+s_{k, n+1}+1}{2}\right) \\
& \cdot \prod_{i<j} \Gamma\left(-s_{i j}\right)
\end{aligned}
$$

namely we have the following integral representation:

$$
\frac{2^{n} \Gamma\left(\frac{n+1}{2}+1\right) D V}{n+1}=\left(\frac{1}{2 \pi i}\right)^{n(n+1) / 2} \int_{r} \hat{V}(s) \prod_{i<j}\left(-2 a_{i j}\right)^{s_{i j}} \prod_{1 \leqq i<j \leqq n+1} d s_{i j}
$$

where $r$ denotes a chain of $n(n+1) / 2$ dimension which is the product of paths $\gamma_{i j}$ defined on each $s_{i j}$-plane as in the figure:

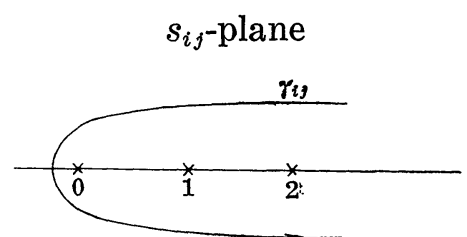

Proof. The integral on each $\gamma_{i j}$ is equal to the sum of all residues on $s_{i j}=0,1,2,3, \cdots$ which gives the power series expansion (4.5).

It is easy to see that $D \cdot V$ is a hypergeometric function of $M-S$ type in the variables $a_{i j}^{2}$. 
Finally some problems unknown to the author are raised here.

Problem 1. To determine all meromorphic 1-forms on $\hat{\mathscr{X}}$ with logarithmic poles along $\bigcup_{1 \leqslant \mu \leqslant \nu} \bigcup_{\left(i_{1} i_{2} \ldots i_{2 \mu-1}\right)} \pi^{-1}\left(\mathfrak{X}_{i_{1} i_{2} \ldots i_{2 \mu-1}}\right)$ and the infinity. It is seen by residue calculus that $2^{n-3} \cdot n(n+1)$ such 1 -forms of the type (3.11) are linearly independent over $\boldsymbol{C}$. For the further properties of logarithmic poles see [7] and [9].

PROBLEM 2. What kind of functions are the inverse of hyperlogarithmic functions? They could be a generalization of exponential functions which satisfy some kind of addition formula and are related to A. N. Parsin's generalized Jacobian variety (see [12] and [13]).

Problem 3. To determine the order of the maximally overdetermined system of (pseudo-) differential equations (6.3).

\section{REFERENCES}

[1] K. Aomoto, Les équations aux différences linéaires et les intégrales des fonctions multiformes, J. Fac. Sci. Univ. Tokyo, 22 (1975), 271-297.

[ 2 ] — Une remarque sur la solution des équations de Schlesinger et Lapp-Danilevski II (to be submitted to J. Fac. Sci. Univ. Tokyo).

[ 3 ] J. Cheeger and J. Simons, Differential characters and geometric invariants (preprint).

[ 4 ] K. T. Chen, Iterated integrals of differential forms and loop space homology, Ann. of Math. 97 (1973), 217-246.

[ 5 ] — Iterated integrals, Fundamental groups and Covering Spaces, Trans. Amer. Math. Soc. 206 (1975), 83-98.

[6] H. S. M. Coxeter, The functions of Schläfli and Lobatschefsky, Quart. J. Math. 6 (1935), 13-29.

[ 7 ] P. Deligne, Théorie de Hodge II, Publ. Math. I.H.E.S. 40 (1971), 5-58.

[ 8 ] H. Hopf, Die curvatura integra Clifford-Kleinscher Raumformen, Nach. v.d. Ges. d. Wiss., Gottingen, Mathem-physik. K.L. 1925.

[9] S. Iitaka, Logarithmic forms of algebraic varieties, J. Fac. Sci. Univ. Tokyo 23 (1976), 525-544.

[10] H. Kneser, Der Simplexinhalt in der nichteuklidischen Geometrie, Deutsch. Math. 1 (1936), 337-340.

[11] H. Poincaré, Sur la généralisation d'un théorème élémentaire de géométrie, Comptes rendus T. 140 (1905), 113-117.

[12] W. Maier und A. Effenberger, Additive Inhaltmasse im positiv gekrümmten Raum, Aeq. Math. 2 (1968), 304-318.

[13] A. N. Parsin, A generalization of Jacobian variety, Izv. Akad. Nauk. SSSR Ser. Mat. 30 (1966), 175-182.

[14] M. Sato, Singular orbits in prehomogeneous vector spaces, Lec. Notes at Univ. of Tokyo, 1972. 
[15] L. Schläfli, On the multiple integral $\iint \cdots \int d x d y \cdots d z$ whose limits are $p_{1}=a_{1} x$ $+b_{1} y+\cdots+h z>0, p_{2}>0, \cdots, p_{n}>0$, and $x^{2}+y^{2}+\cdots+z^{2}<1$, Quart. J. of Math. 3 (1860), 54-68, 97-108.

\section{University of Tokyo}

